\title{
Effects of size on the electrical and optical properties of InGaN-based red light-emitting diodes $\mathbb{P}$
}

Cite as: Appl. Phys. Lett. 116, 173501 (2020); doi: 10.1063/5.0006910

Submitted: 7 March 2020 • Accepted: 14 April 2020 •

Published Online: 27 April 2020

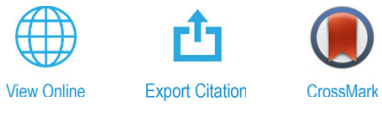

Zhe Zhuang, (D) Daisuke lida, (D) and Kazuhiro Ohkawa ${ }^{\text {a) }}$ (D)

\begin{abstract}
AFFILIATIONS
Computer, Electrical and Mathematical Sciences and Engineering (CEMSE) Division, King Abdullah University of Science and Technology (KAUST), Thuwal 23955-6900, Saudi Arabia
\end{abstract}

a) Author to whom correspondence should be addressed: kazuhiro.ohkawa@kaust.edu.sa

\begin{abstract}
We investigated the effects of size on electrical and optical properties of InGaN-based red light-emitting diodes (LEDs) by designing rectangular chips with different mesa lengths. Larger chips exhibited lower forward voltages because of their lower series resistances. A larger chip helped to realize a longer emission wavelength, narrower full-width at half maximum, and higher external quantum efficiency. However, temperature-dependent electroluminescence measurements indicated that larger chips are detrimental to applications where high temperature tolerance is required. In contrast, a smaller red LED chip achieved a high characteristic temperature of $399 \mathrm{~K}$ and a small redshift tendency of $0.066 \mathrm{~nm} \mathrm{~K}^{-1}$, thus showing potential for temperature tolerant lighting applications.
\end{abstract}

(C) 2020 Author(s). All article content, except where otherwise noted, is licensed under a Creative Commons Attribution (CC BY) license (http:// creativecommons.org/licenses/by/4.0/). https://doi.org/10.1063/5.0006910

InGaN-based blue/green light-emitting diodes (LEDs) have become increasingly prevalent in illumination and display applications, such as interior/exterior lighting and display backlights. ${ }^{1-}$ However, their performance declines rapidly with the emission wavelength, especially in the red region. ${ }^{4,5}$ This decline is related to the content of In in InGaN quantum wells (QWs). InGaN layers with a high In content have a high density of defects and a strong quantumconfined Stark effect (QCSE).

Much effort has been devoted to improving InGaN-based red LEDs with approaches including InGaN quantum dots, ${ }^{8}$ a semipolar InGaN buffer layer, ${ }^{9}$ lattice-matched $\mathrm{InGaN}_{\mathrm{ScAlMgO}}(0001)$ templates, ${ }^{10}$ and InGaN/GaN nanowires. ${ }^{11,12}$ Our high-temperature InGaN growth technique realized deep-red electroluminescence (EL) with 740-nm emission. ${ }^{13,14}$ We have also proposed several straincompensation approaches to reduce defects, such as AlN/AlGaN barriers, ${ }^{15}$ hybrid QW structures, ${ }^{16}$ and different thicknesses of $\mathrm{GaN}$ templates. ${ }^{17}$ Recently, a very low forward voltage of $2.45 \mathrm{~V}$ at $20 \mathrm{~mA}$ was demonstrated in a $665-\mathrm{nm}$ InGaN-based red LED on a $\beta-\mathrm{Ga}_{2} \mathrm{O}_{3}$ substrate. $^{18}$

Device design and optimization are also critical for improving the performance of InGaN-based red LEDs. Previous works have demonstrated that chip size has a significant influence on the efficiency droop of InGaN-based blue LEDs. ${ }^{19-22}$ Different chip sizes result in different temperature tolerance. ${ }^{19}$ LEDs ranging from the micro-scale to large sizes have shown the current crowding effect and carrier recombination on the edge surfaces of the device. ${ }^{20}$ However, InGaNbased red LEDs have not been studied and optimized as much as blue ones, which have a long history.

This work examines the effect of size on device performance using different rectangular chips on the same InGaN-based red LED wafer. Current-voltage $(I-V)$ curves were used to characterize the forward voltages and reverse leakage currents. We systematically investigated the EL characteristics at different currents, including the peak wavelength, full-width at half-maximum (FWHM), external quantum efficiency (EQE), and integrated intensity. The results demonstrate that the chip size can be adjusted to control the performance of InGaN-based red LEDs.

InGaN-based red LED epitaxial wafers were grown on $c$-plane patterned sapphire substrates by metalorganic vapor-phase epitaxy (MOVPE) in a single-wafer horizontal reactor at $100 \mathrm{kPa}$. The epitaxial structures of the InGaN-based red LEDs were reported in previous works. ${ }^{15-17}$ We fabricated red LEDs using our standard processes. Indium tin oxide (ITO) films were deposited as a transparent conductive layer and subjected to two-step annealing to form Ohmic contacts with $\mathrm{p}-\mathrm{GaN}$. $^{23}$ LED mesas were obtained by standard photolithography and inductively coupled plasma etching to expose the n-type layer. 


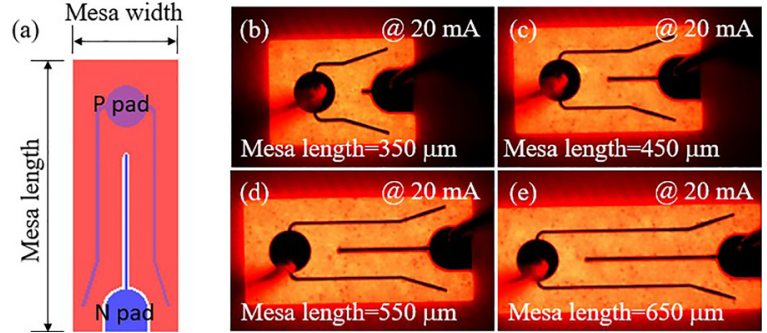

FIG. 1. (a) LED chip design. EL images of red LEDs with the mesa lengths of (b) $350 \mu \mathrm{m}$, (c) $450 \mu \mathrm{m}$, (d) $550 \mu \mathrm{m}$, and (e) $650 \mu \mathrm{m}$ at $20 \mathrm{~mA}$.

Finally, electrodes based on $\mathrm{Cr} / \mathrm{Ni} / \mathrm{Au}(50 \mathrm{~nm} / 20 \mathrm{~nm} / 200 \mathrm{~nm})$ were deposited on both ITO and n-type layers as contact pads.

Electrical pumping of all red LED chips was carried out at a probe station using a semiconductor parameter analyzer. The EL characteristics were measured at temperatures ranging from $295 \mathrm{~K}$ (room temperature, RT) to $373 \mathrm{~K}$. The temperatures were measured from the sample-holding stage of the probe station.

Figure 1(a) shows the pattern of the rectangular LED chips. The chips had a constant mesa width of $250 \mu \mathrm{m}$, while the mesa lengths were $350 \mu \mathrm{m}, 450 \mu \mathrm{m}, 550 \mu \mathrm{m}$, and $650 \mu \mathrm{m}$. Thus, the active areas were calculated to be $0.0755 \mathrm{~mm}^{2}, 0.0985 \mathrm{~mm}^{2}, 0.1215 \mathrm{~mm}^{2}$, and $0.1445 \mathrm{~mm}^{2}$, respectively. The active area is the mesa area of each LED chip. Figures 1(b) -1 (e) show EL images of the red LED chips with different sizes at $20 \mathrm{~mA}$. All LEDs showed homogeneous EL emission, good current spreading, and no significant current crowding. However, many dark spots were observed in the emission area, which might be related to threading dislocations and trench defects. ${ }^{1}$

We studied the electrical properties of LEDs with different mesa lengths. Figure 2(a) shows a typical $I-V$ curve of a red LED with a mesa length of $650 \mu \mathrm{m}$ at RT. The curve at the forward bias displayed a sharp onset voltage and then exponentially increased with the injection current, exhibiting the standard behavior of a $\mathrm{p}-\mathrm{n}$ junction diode. Figure 2(b) shows the forward voltages at $20 \mathrm{~mA}$ as a function of the inverse of the active area. The forward voltages obtained with different mesa lengths were found to scale linearly with the inverse of the active area. LED chips with larger active areas exhibited lower forward voltages because of their lower series resistance originating from the larger geometry. ${ }^{24}$ Therefore, a larger chip can improve the wall-plug efficiency (WPE) of red LEDs. ${ }^{25}$
Figure 2(a) also shows typical reverse currents of the red LED under reverse bias. The reverse current increased with the reverse voltage, which was presumably caused by crystal defects such as V-pits and trench defects. The reverse currents at $-5 \mathrm{~V}$ are plotted against the active area in Fig. 2(c). The average values showed a trend of the reverse currents linearly increasing with the active area. This trend is reasonable because a larger area will have a larger number of defects, which results in current leakage channels. Therefore, higher crystal quality of the red LED structures is necessary to suppress the leakage current.

The EL spectra were measured at $5-100 \mathrm{~mA}$ at RT. The average peak wavelengths and FWHMs are shown in Figs. 3(a) and (b), respectively. Significant blueshifts of approximately $50 \mathrm{~nm}$ were observed between the peak wavelengths at $5 \mathrm{~mA}$ and $100 \mathrm{~mA}$. The amount of blueshift for the red LEDs was much higher than that of the blue or green ones, ${ }^{26,27}$ demonstrating that red LEDs suffer from significant QCSE.

The peak wavelengths of smaller LEDs had blueshifts compared to those of larger ones at the same currents. This means that a higher current density accelerates the screening of QCSE by free carriers. The LED size is also crucial for the peak wavelength in high-In-content InGaN-based red LEDs, and so we must consider the In content in QWs and the chip size together. Generally, the fundamental transition energy of a QW could be reduced by increasing the In content or decreasing the screening of the internal electric field in a QW. ${ }^{7}$ When designing red QWs with a fixed thickness for a particular red emission wavelength at a specific operating current, larger chips need a lower In content in the active regions than those of smaller chips because larger chips exhibit less screening of the internal electric field (QCSE) due to a lower current density. Since the lower In content has a merit of maintaining the crystal qualities of the LED structures, a larger chip is preferable for better device performance.

Interestingly, the peak wavelengths were identical for different chip sizes at the same current density, which means that the amount of QCSE only depends on the current density. Therefore, our device fabrication did not influence the residual strain and localized states in the InGaN QWs. This situation differs from those of nanostructurebased LEDs with partially relaxed InGaN QWs. ${ }^{26}$

The FWHMs dropped quickly to the minimum values (approximately $60 \mathrm{~nm}$ ) in the low current range in Fig. 3(b). All chip sizes indicated the same behavior, which was similar to that in previous reports. ${ }^{5,15,16}$ The FWHMs of smaller chips were narrower than those
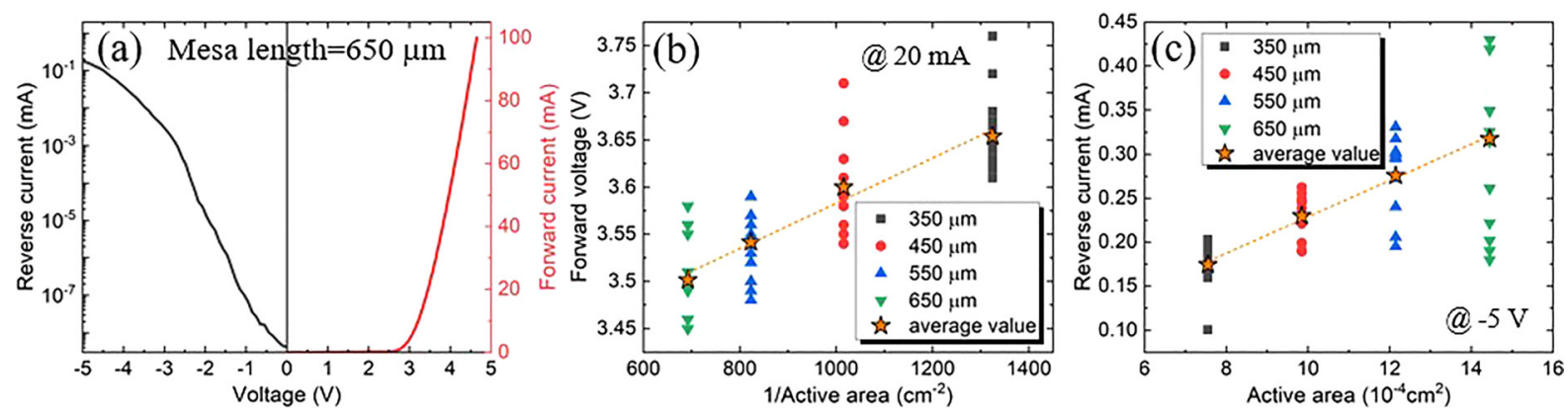

FIG. 2. (a) $I-V$ characteristics of an LED device with a mesa length of $650 \mu \mathrm{m}$. (b) The forward voltage of 10 LED devices with different sizes as a function of $1 /$ active area at $20 \mathrm{~mA}$. (c) Reverse current of 10 LED devices with different sizes as a function of the active area at $-5 \mathrm{~V}$. The stars represent the average values of 10 devices, and the dashed lines are the linear fit of the average values. 

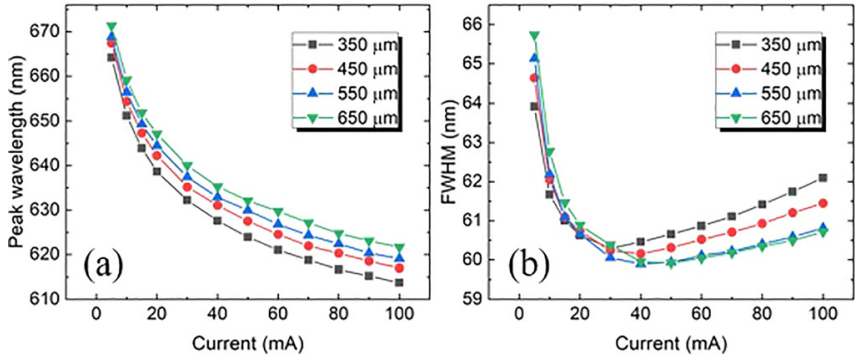

FIG. 3. Current dependencies of (a) peak wavelengths and (b) FWHMs. Each point is an average from over 10 LEDs for each mesa length.

of larger chips at low current below $20 \mathrm{~mA}$, which is attributed to the rapid saturation of the emission from deep localized states in the InGaN active region for the smaller chips. The minimum values depended on the LED chip size, and a larger chip had a smaller value.

After the minimum values, each FWHM increased with the current. The size dependencies of the minimum FWHM and the FWHM increment originated from the effect of heat generation ${ }^{28}$ on the InGaN active region. The current densities of smaller chips are higher than those of larger chips, and so the temperatures of smaller ones will be higher, resulting in the continuous increment of FWHM after the minimum value. In contrast, heat generation in a larger LED can be suppressed. Thus, the FWHM of $650-\mu \mathrm{m}$ chips had the smallest minimum around $60 \mathrm{~nm}$ and increased more slowly than those of smaller chips.

We operated a $650-\mu$ m chip under pulsed operation with a duty cycle of $20 \%$. The results indicated a constant FWHM of approximately $59 \mathrm{~nm}$ at currents of $40-100 \mathrm{~mA}$. Robin et al. reported that the FWHM is vital to achieving pure red emission. ${ }^{29}$ Therefore, fabricating larger chips is recommended because they are expected to have narrower FWHMs.

We also investigated the effects of the size on the EL integrated intensity of red LEDs. Figures 4(a)-4(c) show the EL integrated intensities and their peak wavelengths at currents of $20 \mathrm{~mA}, 30 \mathrm{~mA}$, and $60 \mathrm{~mA}$. At $20 \mathrm{~mA}$ [Fig. 4(a)], the LEDs with shorter mesa lengths exhibited higher EL intensities and shorter peak wavelengths. However, the LEDs with longer mesa lengths indicated higher EL intensities at $30 \mathrm{~mA}$ and $60 \mathrm{~mA}$. These results are attributed to the current density and the efficiency droop, as shown in Fig. 4(d).

All the maximum EQE values occurred around $26 \mathrm{~A} / \mathrm{cm}^{2}$. The current density was just $26 \mathrm{~A} / \mathrm{cm}^{2}$ for the smallest chips with a mesa length of $350 \mu \mathrm{m}$ at $20 \mathrm{~mA}$, while those of the other chips were below $26 \mathrm{~A} / \mathrm{cm}^{2}$. Therefore, the smaller chips showed higher EL intensities due to the higher EQEs. At $30 \mathrm{~mA}$, the current density of the chips with a mesa length of $550 \mu \mathrm{m}$ was around $26 \mathrm{~A} / \mathrm{cm}^{2}$ at the EQE maxima. Thus, the EQEs of all chips were located around the maximum, leading to a similar EL intensity profile to the EQE profile.

At $60 \mathrm{~mA}$, the current densities of all chips exceeded $26 \mathrm{~A} / \mathrm{cm}^{2}$. Therefore, the larger chips with lower current densities showed higher EL intensities. The inset also shows that the maximum EQE values were higher for the larger chips, indicating that a larger chip has a merit of realizing a higher EQE value.

Generally, the carrier loss due to surface recombination increases with the decreasing chip size down to micro-LEDs, ${ }^{20,22}$ which causes a

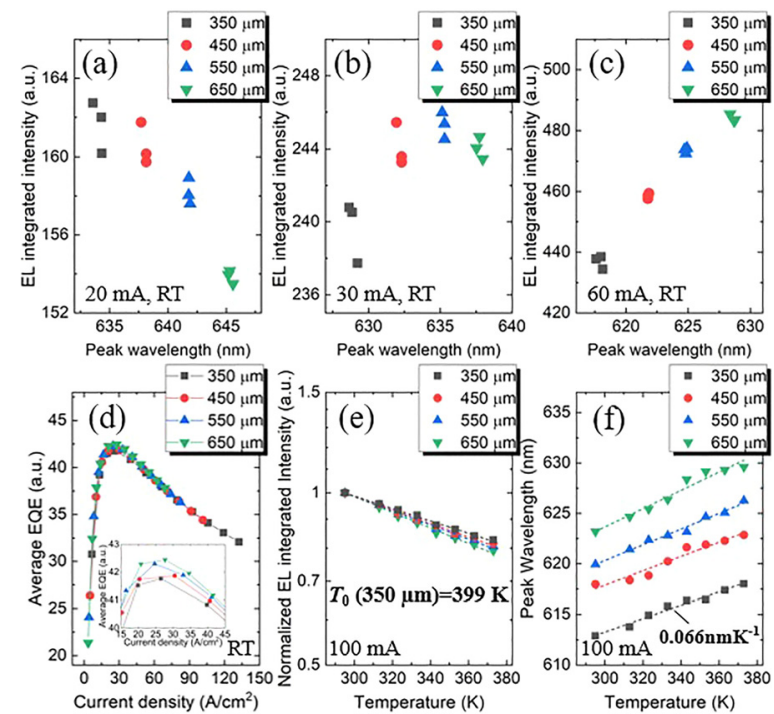

FIG. 4. EL integrated intensities and peak wavelengths obtained at the currents of (a) $20 \mathrm{~mA}$, (b) $30 \mathrm{~mA}$, and (c) $60 \mathrm{~mA}$ for three LEDs with four different mesa lengths. (d) Current-density dependencies of the average EQEs obtained from three LEDs with different mesa lengths. The inset shows the area around the EQE maxima. Temperature dependencies of (e) the normalized EL integrated intensities and (f) the peak wavelengths of LEDs with different mesa lengths at $100 \mathrm{~mA}$.

significant decrease in EQE, even at the same current density. However, the EQE values in Fig. 4(d) were found to be independent of the chip sizes at the same current density except for the maximum EQE values. This indicates that the electron-hole recombination on the surface can be almost negligible with these relatively large chip sizes.

Figure 4(e) shows the temperature dependence of the normalized EL integrated intensity from $295 \mathrm{~K}$ (RT) to $373 \mathrm{~K}$. The LED chips were operated at a constant current of $100 \mathrm{~mA}$. The EL integrated intensities dropped down with the temperatures for all LEDs, which is usually called the thermal droop. An LED with a shorter mesa length exhibited a smaller temperature dependence of the EL intensity although the LED had the lowest EQEs at $100 \mathrm{~mA}$. The thermal droop of the EL intensity is described by

$$
I=I_{T=295 K} \exp \left(-\frac{T-295 K}{T_{0}}\right),
$$

where $I$ is the normalized EL integrated intensity, $I_{T=295 \mathrm{~K}}$ is the EL integrated intensity at $295 \mathrm{~K}, T[\mathrm{~K}]$ is the stage temperature, and $T_{0}$ $[\mathrm{K}]$ is the characteristic temperature.

The characteristic temperature is a standard quantitative parameter to evaluate the temperature dependence. A larger characteristic temperature implies a weaker temperature dependence. From the fitting of the experimental results, the values of $T_{0}$ were measured to be $399 \mathrm{~K}, 367 \mathrm{~K}, 348 \mathrm{~K}$, and $319 \mathrm{~K}$ for the chips with mesa lengths of $350 \mu \mathrm{m}, 450 \mu \mathrm{m}, 550 \mu \mathrm{m}$, and $650 \mu \mathrm{m}$, respectively. The reduction behavior of $T_{0}$ with the increasing mesa length is due to Shockley-Read-Hall (SRH) recombination. ${ }^{19}$ SRH recombination becomes effective at higher temperature but can be suppressed at higher current density. 
Therefore, the EL integrated intensities dropped as the temperature increased, but less so at higher current densities. Consequently, the smallest chips, which worked at the highest current density, showed the highest characteristic temperature compared to other larger chips. The characteristic temperature was $399 \mathrm{~K}$ at $100 \mathrm{~mA}$ for the smallest chip, which is comparable to those reported for InGaNbased green LEDs ${ }^{30}$ and AlGaInP-based red LEDs. ${ }^{31,32}$

Figure $4(\mathrm{f})$ shows the temperature dependence of the peak wavelength at a constant current of $100 \mathrm{~mA}$. Redshifts were observed at the peak wavelengths for all chips as the temperature changed. The redshift coefficients from the linear fittings were $0.066 \mathrm{~nm} \mathrm{~K}^{-1}, 0.071 \mathrm{~nm} \mathrm{~K}^{-1}$, $0.078 \mathrm{~nm} \mathrm{~K}^{-1}$, and $0.091 \mathrm{~nm} \mathrm{~K}^{-1}$ for the chips with mesa lengths of $350 \mu \mathrm{m}, 450 \mu \mathrm{m}, 550 \mu \mathrm{m}$, and $650 \mu \mathrm{m}$, respectively. These data indicate that smaller chips had smaller redshift coefficients. This tendency can be explained by the energy distribution of carriers. ${ }^{3}$

The bandgap of InGaN shrunk as the temperature increased, which caused the redshift of the peak wavelengths for all chips. Meanwhile, carriers in QWs could move to higher energy levels with the increasing temperature, which conversely resulted in the blueshift of the peak wavelength for all chips. ${ }^{33}$ Because smaller chips had a higher current density (carrier density), carriers in smaller chips would have a higher transition probability of moving to higher energy levels. As a result, more percentages of carriers in smaller chips would move to higher energy levels, making the energy distribution of carriers higher than that in larger chips. The higher energy distribution of carriers in smaller chips would make the peak wavelength show a larger blueshift, and the larger blueshift could compensate more for the redshift due to the bandgap shrinking. Therefore, we finally observed the smallest redshift coefficient from the smallest chip. In the case of AlInGaP-based red LEDs, the redshift coefficients for the chip sizes of $300 \times 300 \mu \mathrm{m}^{2}$ and $500 \times 500 \mu \mathrm{m}^{2}$ were measured to be $0.142 \mathrm{~nm} \mathrm{~K}^{-1}$ and $0.148 \mathrm{~nm} \mathrm{~K}^{-1}$ at a pulsed current of $100 \mathrm{~mA}$, respectively. ${ }^{31,32}$ These redshift coefficients were almost twice of that for our smallest chip, demonstrating that InGaN-based red LEDs are proper candidates for temperature tolerant lighting applications.

In summary, we investigated the effects of size on the performance of InGaN-based red LEDs by designing rectangular chips with different mesa lengths on the same LED wafer. Larger chips exhibited lower forward voltages at $20 \mathrm{~mA}$ due to their lower series resistance originated from the device area. Furthermore, a larger chip resulted in a longer emission wavelength, narrower FWHM, and higher EQE at high currents. These characteristics are beneficial for InGaN-based red LEDs. On the other hand, smaller chips had merits of a high characteristic temperature of $399 \mathrm{~K}$ and a small redshift coefficient of $0.066 \mathrm{~nm} \mathrm{~K}^{-1}$. These data suggest a potential for temperature tolerant lighting applications by reducing the size of InGaN-based red chips.

This work was financially supported by the King Abdullah University of Science and Technology (KAUST) (No. BAS/1/1676-01-01).

The data that support the findings of this study are available within this article.

\section{REFERENCES}

'Z. Zhuang, X. Guo, B. Liu, F. Hu, Y. Li, T. Tao, J. Dai, T. Zhi, Z. Xie, P. Chen, D. Chen, H. Ge, X. Wang, M. Xiao, Y. Shi, Y. Zheng, and R. Zhang, Adv. Funct. Mater. 26, 36 (2016).
${ }^{2}$ B. Liu, D. Chen, H. Lu, T. Tao, Z. Zhuang, Z. Shao, W. Xu, H. Ge, T. Zhi, F. Ren, J. Ye, Z. Xie, and R. Zhang, "Hybrid light emitters and UV solar-blind avalanche photodiodes based on III-nitride semiconductors," Adv. Mater. (published online, 2020).

${ }^{3}$ Y. Narukawa, M. Ichikawa, D. Sanga, M. Sano, and T. Mukai, J. Phys. D: Appl. Phys. 43, 354002 (2010).

${ }^{4}$ F. Y. Jiang, J. L. Zhang, L. Q. Xu, J. Ding, G. X. Wang, X. M. Wu, X. L. Wang, C. L. Mo, Z. J. Quan, X. Guo, C. D. Zheng, S. Pan, and J. L. Liu, Photonics Res. 7, 144 (2019).

5J. I. Hwang, R. Hashimoto, S. Saito, and S. Nunoue, Appl. Phys. Express 7, 071003 (2014).

${ }^{6}$ A. Vaitkevičius, J. Mickevičius, D. Dobrovolskas, Ö. Tuna, C. Giesen, M. Heuken, and G. Tamulaitis, J. Appl. Phys. 115, 213512 (2014).

${ }^{7}$ B. Damilano and B. Gil, J. Phys. D: Appl. Phys. 48, 403001 (2015).

${ }^{8}$ W. Lv, L. Wang, J. Wang, Y. Xing, J. Zheng, D. Yang, Z. Hao, and Y. Luo, Jpn. J. Appl. Phys., Part 1 52, 08JG13 (2013).

${ }^{9}$ I. L. Koslow, M. T. Hardy, P. S. Hsu, P.-Y. Dang, F. Wu, A. Romanov, Y.-R. Wu, E. C. Young, S. Nakamura, J. S. Speck, and S. P. DenBaars, Appl. Phys. Lett. 101, 121106 (2012).

${ }^{10}$ T. Ozaki, M. Funato, and Y. Kawakami, Appl. Phys. Express 12, 011007 (2019).

${ }^{11}$ M. R. Philip, D. D. Choudhary, M. Djavid, K. Q. Le, J. Piao, and H. P. T. Nguyen, J. Sci.: Adv. Mater. Devices 2, 150 (2017).

${ }^{12}$ Y. Y. Ou, D. Iida, J. Liu, K. Y. Wu, K. Ohkawa, A. Boisen, P. M. Petersen, and H. Y. Ou, Nanophotonics 7, 317 (2018).

${ }^{13}$ K. Ohkawa, T. Watanabe, M. Sakamoto, A. Hirako, and M. Deura, J. Cryst. Growth 343, 13 (2012).

${ }^{14}$ K. Ohkawa, F. Ichinohe, T. Watanabe, K. Nakamura, and D. Iida, J. Cryst. Growth 512, 69 (2019).

${ }^{15}$ D. Iida, S. Lu, S. Hirahara, K. Niwa, S. Kamiyama, and K. Ohkawa, J. Cryst. Growth 448, 105 (2016).

${ }^{16}$ D. Iida, K. Niwa, S. Kamiyama, and K. Ohkawa, Appl. Phys. Express 9, 111003 (2016).

${ }^{17}$ D. Iida, Z. Zhuang, P. Kirilenko, M. Velazquez-Rizo, M. Najmi, and K. Ohkawa, Appl. Phys. Lett. 116, 162101 (2020).

${ }^{18}$ D. Iida, Z. Zhuang, P. Kirilenko, M. Velazquez-Rizo, and K. Ohkawa, Appl. Phys. Express 13, 031001 (2020).

${ }^{19}$ D. S. Meyaard, Q. Shan, J. Cho, E. F. Schubert, S.-H. Han, M.-H. Kim, C. Sone, S. J. Oh, and J. K. Kim, Appl. Phys. Lett. 100, 081106 (2012).

${ }^{20}$ S. S. Konoplev, K. A. Bulashevich, and S. Y. Karpov, Phys. Status Solidi A 215, 1700508 (2018).

${ }^{21}$ S.-C. Huang, H. Li, Z.-H. Zhang, H. Chen, S.-C. Wang, and T.-C. Lu, Appl. Phys. Lett. 110, 021108 (2017).

${ }^{22}$ M. S. Wong, C. Lee, D. J. Myers, D. Hwang, J. A. Kearns, T. Li, J. S. Speck, S. Nakamura, and S. P. DenBaars, Appl. Phys. Express 12, 097004 (2019).

${ }^{23}$ Z. Zhuang, D. Iida, P. Kirilenko, M. Velazquez-Rizo, and K. Ohkawa, Opt. Express 28, 12311 (2020).

${ }^{24}$ N. L. Ploch, H. Rodriguez, C. Stolmacker, M. Hoppe, M. Lapeyrade, J. Stellmach, F. Mehnke, T. Wernicke, A. Knauer, V. Kueller, M. Weyers, S. Einfeldt, and M. Kneissl, IEEE Trans. Electron Devices 60, 782 (2013).

${ }^{25}$ L. Y. Kuritzky, C. Weisbuch, and J. S. Speck, Opt. Express 26, 16600 (2018).

${ }^{26}$ Z. Zhuang, X. Guo, B. Liu, F. Hu, J. Dai, Y. Zhang, Y. Li, T. Tao, T. Zhi, Z. Xie, H. Ge, X. Wang, M. Xiao, T. Wang, Y. Shi, Y. Zheng, and R. Zhang, Nanotechnology 27, 015301 (2016).

${ }^{27}$ S. Huang, Z. Chen, Y. Xian, B. Fan, Z. Zheng, Z. Wu, H. Jiang, and G. Wang, Appl. Phys. Lett. 101, 041116 (2012).

${ }^{28}$ M. Funato, M. Ueda, Y. Kawakami, Y. Narukawa, T. Kosugi, M. Takahashi, and T. Mukai, Jpn. J. Appl. Phys., Part 2 45, L659 (2006).

${ }^{29}$ Y. Robin, M. Pristovsek, H. Amano, F. Oehler, R. A. Oliver, and C. J. Humphreys, J. Appl. Phys. 124, 183102 (2018).

${ }^{30}$ S. H. Lee, H. K. Lee, S. Bae, J. Kim, S.-B. Kim, and J. S. Yu, Phys. Status Solidi A 210, 2479 (2013).

${ }^{31}$ M. S. Kim, H. K. Lee, and J. S. Yu, Semicond. Sci. Technol. 28, 025005 (2013).

${ }^{32}$ H. K. Lee, D. H. Lee, Y. M. Song, Y. T. Lee, and J. S. Yu, Solid-State Electron. 56, 79 (2011)

${ }^{33}$ T. Mukai, M. Yamada, and S. Nakamura, Jpn. J. Appl. Phys., Part 2 37, L1358 (1998). 